\title{
Plasma-Erosion Properties of Ceramic Coating Prepared by Plasma Spraying
}

\author{
Junya Kitamura, Hiroaki Mizuno, Nobuaki Kato and Isao Aoki \\ Thermal Spray Materials Department, Fujimi Incorporated, Kakamigahara 509-0103, Japan
}

Application of plasma sprayed ceramic coatings with a high purity of more than $99.9 \%$ has been sharply increasing in semiconductor and liquid-crystal-display (LCD) production equipments in the last few years. The size of the equipments becomes larger with increasing $\mathrm{Si}$ wafer size and the LCD size that promotes the replacement from conventional techniques, such as alumite film and bulk ceramics, to plasma spray coatings, where the high durability against the plasma erosion (anti-plasma erosion resistance) is required. However, as far as we know, no systematic studies on the plasma-erosion properties are reported. In this work, durability of plasma sprayed alumina and yttria coatings against $\mathrm{CF}_{4} / \mathrm{O}_{2}$ plasma are investigated by reactive ion etching (RIE) system and are compared to that of the conventional techniques. The erosion mechanism and the effect of the powder properties are discussed through the micro-structural analysis. [doi:10.2320/matertrans.47.1677]

(Received December 26, 2005; Accepted April 13, 2006; Published July 15, 2006)

Keywords: plasma spray, ceramic coating, alumina, yttria, erosion, semiconductor equipment

\section{Introduction}

The size of semiconductor and liquid-crystal-display (LCD) production equipments for dry etching, sputtering and ashing has been increasing due to increasing the size of Si wafer (from 200 to $300 \mathrm{~mm}$ in diameter) and of the LCD, where plasma treatment is effectively used for micro fabrication. Up to now, anodizing of aluminum alloy (alumite film) are mainly used as a shield to protect the chamber parts from the plasma, which intensely erodes the parts mainly composed of aluminum or stainless steel, resulting in both frequent maintenance and decrease of yield ratio of the $\mathrm{Si}$ device and LCD device that seriously affect cost increase. In addition, bulk ceramics, such as alumina, silicon carbide and silicon nitride are mainly used as an electrostatic chuck (ESC) to stabilize the $\mathrm{Si}$ wafer and the LCD during fabrication process. Recently, increase of the plasma power by increasing the wafer and the LCD size caused unreliability of the alumite film because of its insufficient durability against the high power processing plasma of $\mathrm{CF}_{4}, \mathrm{SF}_{6}, \mathrm{O}_{2}$ and $\mathrm{Cl}_{2}$, for example. It also becomes difficult to apply large scaled bulk ceramics as the shield and the ESC due to technical difficulty and high cost in spite of higher durability. ${ }^{1,2)}$ Especially, the damage of the chamber by the processing plasma becomes serious in the dry etching process, where reactive ion etching (RIE) is generally used.

In order to apply to large scaled parts retaining affordable price, utilization of ceramic coatings prepared by plasma spraying for the chamber shield and for the ESC has been sharply increasing in the last few years. ${ }^{3)}$ As a coating material, alumina $\left(\mathrm{Al}_{2} \mathrm{O}_{3}\right)$ has been used presently due to its high dielectric strength and high durability against the processing plasma. ${ }^{4}$ For semiconductor production equipments, alumina coating of more than $99.9 \%$ in purity is generally required to eliminate the influence of impurities on the performance of the devices. Yttria $\left(\mathrm{Y}_{2} \mathrm{O}_{3}\right)$ coatings have been also gradually applied to the chamber shields because these have higher durability against the processing plasma. ${ }^{1,5)}$ However, no systematic investigations about the plasma sprayed ceramic coatings for the ESC and the chamber shield are found as far as we know. In addition, it seems that no reports are found about erosion mechanism of the ceramic coatings, such as alumina, yttria and so on, by the processing plasma that will clarify key factors to improve the current status and problems. In this study, anti-plasma erosion resistance of plasma sprayed ceramic coatings of alumina $\left(\mathrm{Al}_{2} \mathrm{O}_{3}\right)$ and yttria $\left(\mathrm{Y}_{2} \mathrm{O}_{3}\right)$ against $\mathrm{CF}_{4} / \mathrm{O}_{2}$ plasma has been investigated by reactive ion etching (RIE) system and has been compared to that of conventional techniques, such as alumite film and bulk ceramics. ${ }^{6}$ Mechanism of the plasma erosion and the effect of powder properties have been discussed through the micro-structural analysis using scanning electron microscopy (SEM).

\section{Experimental}

\subsection{Spray powders and spray conditions}

The spray powders used in this study are summarized in Table 1. Fused-and-crushed alumina $\left(\mathrm{Al}_{2} \mathrm{O}_{3}\right)$ powders (A1 and A2) were used. Average particle diameter of A2 was smaller than that of A1. As for yttria $\left(\mathrm{Y}_{2} \mathrm{O}_{3}\right)$, agglomeratedand-sintered powders with the purity of more than $99.9 \%$ were used. Primary-particle diameter was changed ranging from 0.6 to $5.3 \mu \mathrm{m}$ (Y1-Y3). Y4 was fused-and-crushed yttria powder and its purity was $99 \%$. Both sintered-bulk alumina (A3) and yttria (Y5) were also used for the plasma

Table 1 Specimens for plasma erosion tests.

\begin{tabular}{|c|c|c|c|c|c|}
\hline Specimens & Material & $\begin{array}{c}\text { Purity } \\
{[\%]}\end{array}$ & $\begin{array}{c}\text { Manufacturing } \\
\text { method }\end{array}$ & $\begin{array}{c}\text { Average } \\
\text { diameter* } \\
{[\mu \mathrm{m}]}\end{array}$ & $\begin{array}{c}\text { Primary-particle } \\
\text { diameter } \\
{[\mu \mathrm{m}]}\end{array}$ \\
\hline $\mathrm{Al}$ & \multirow{3}{*}{$\begin{array}{l}\text { Alumina } \\
\left(\mathrm{Al}_{2} \mathrm{O}_{3}\right)\end{array}$} & 99.9 & \multirow{3}{*}{$\begin{array}{l}\text { Fused-and- } \\
\text { crushed }\end{array}$} & 29.6 & \multirow{3}{*}{ - } \\
\hline A2 & & 99.9 & & 25.8 & \\
\hline A3 & & 99.9 & & Sintered-bulk & \\
\hline Y1 & \multirow{5}{*}{$\begin{array}{c}\text { Yttria } \\
\left(\mathrm{Y}_{2} \mathrm{O}_{3}\right)\end{array}$} & 99.9 & \multirow{3}{*}{$\begin{array}{l}\text { Agglomerated- } \\
\text { and-sintered }\end{array}$} & 39.0 & 0.6 \\
\hline Y2 & & 99.9 & & 36.3 & 2.9 \\
\hline Y3 & & 99.9 & & 42.8 & 5.3 \\
\hline Y4 & & 99 & $\begin{array}{l}\text { Fused-and- } \\
\text { crushed }\end{array}$ & 39.9 & - \\
\hline Y5 & & 99 & & Sintered-bulk & \\
\hline
\end{tabular}

*: The diameter of agglomerated and sintered powder for $\mathrm{Y} 1,2$ and 3. 

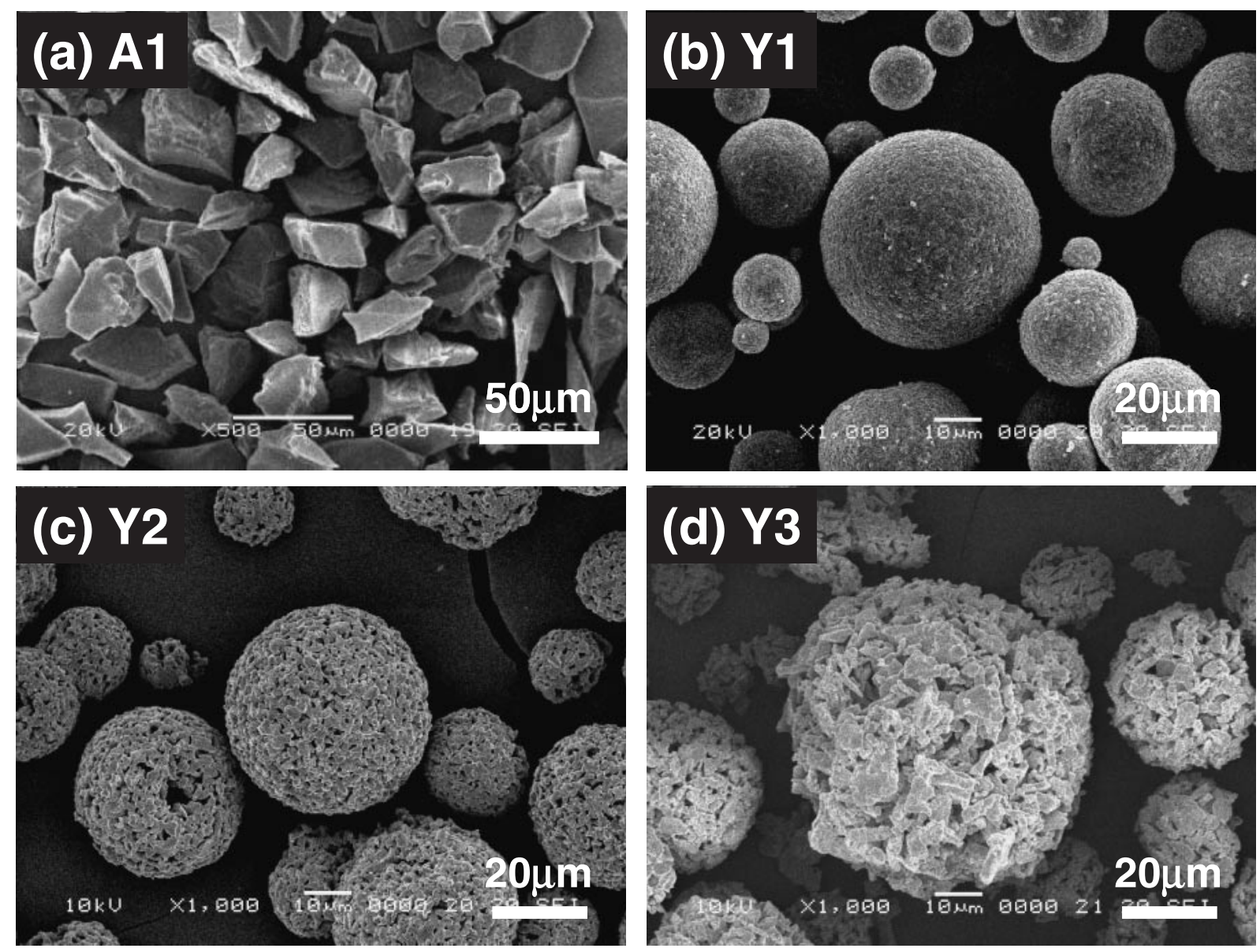

Fig. 1 SEM images of the spray powders of alumina (A1) and agglomerated-and-sintered yttria (Y1-Y3). The average diameters of the primary-particle of yttria are (b) 0.6 , (c) 2.9 and (d) $5.3 \mu \mathrm{m}$, respectively.

Table 2 Spray conditions.

\begin{tabular}{ccc}
\hline Spray equipment & \multicolumn{2}{c}{ Conditions } \\
\hline Gun: SG-100 (40 kW) & Ar pressure (MPa) & 0.34 \\
Feeder: Model 1264 & He pressure (MPa) & 0.34 \\
(Praxair) & Spray distance $(\mathrm{mm})$ & 120 \\
\hline
\end{tabular}

erosion test as a reference, whose sizes were $15 \times 15 \times$ $5.0^{t} \mathrm{~mm}$. The alumina (A1 and A2) powders and agglomerated-and-sintered yttria powders (Y1-Y3) have been developed by Fujimi Incorporated (Japan). SEM images of the spray powders of $\mathrm{A} 1$ and $\mathrm{Y} 1-\mathrm{Y} 3$ are shown in Fig. 1. Fused-and-crushed powder of yttria (Y4) has been also prepared by Fujimi Incorporated as a reference because its lower purity is difficult to apply to semiconductor production equipments. Sintered-bulk ceramics were prepared by Tsukuba Ceramic Works (Japan).

Spray conditions are summarized in Table 2. Conventional atmospheric plasma spraying was conducted to prepare the coatings. Substrates of aluminum alloy (A6061) with the size of $50 \times 70 \times 5.0^{t} \mathrm{~mm}$, were sand blasted by alumina grit \#40 before plasma spraying. The coating thickness was set to $200 \mu \mathrm{m}$. The colors of the coatings after plasma spraying were pure white except for Y1, whose color was changed to deep pink from the white powder. The color of yttria coatings tended to become pink with decreasing primary-particle size from Y3 to Y1. Porosity of all spray coatings was estimated to be almost $5 \%$ by imaging analysis. Within yttria coatings, pore size of the Y3 (larger primary particle size) coating tended to be larger. As for another coating structures, such as inclusion of unmolten particles and lamellar structure, the difference between the coatings is unclear within optical microscopic analysis. The sprayed specimens were cut with the size of $15 \times 15 \mathrm{~mm}$ after spraying. Surfaces of sprayed coatings and sintered-bulk ceramics were mirror-polished using colloidal silica with an average diameter of $0.06 \mu \mathrm{m}$ to study both the durability against the processing plasma and the erosion mechanism in detail.

\subsection{Conditions of the plasma erosion tests}

Conditions of the plasma erosion tests are summarized in Table 3. In order to investigate the effect of the plasma power on the durability of the specimens, the erosion tests at low $(80 \mathrm{~W})$, medium $(175 \mathrm{~W})$ and high $(800 \mathrm{~W})$ plasma power conditions were conducted. As for the low power, the erosion (etching) rate of silicon dioxide $\left(\mathrm{SiO}_{2}\right)$ onto $\mathrm{Si}$ wafer is 50 $\mathrm{nm} / \mathrm{min}$. The RIE systems used in this study were Model RIE-200L (Samco Inc. Japan) for low and medium power and NLD-800 (ULVAC Inc. Japan) for high plasma power. Effective exposure area of the processing plasma in the both systems was $200 \mathrm{~mm}$ in diameter. Before introducing the RIE chambers, the surface of the mirror-polished specimens was masked by polyimide tape partially. The erosion rate was 
Table 3 Conditions of the plasma erosion tests.

\begin{tabular}{|c|c|c|c|}
\hline Parameters & Low & Medium & High \\
\hline $\mathrm{CF}_{4}$ flow rate $(\mathrm{L} / \mathrm{min})$ & & 0.054 & \\
\hline $\mathrm{O}_{2}$ flow rate $(\mathrm{L} / \mathrm{min})$ & & 0.005 & \\
\hline Chamber pressure $(\mathrm{Pa})$ & & 5 & 1 \\
\hline Plasma power (W) & 80 & 175 & 800 \\
\hline Exposure area (mm) & & 200 & \\
\hline Exposure time (min) & & 480 & 60 \\
\hline
\end{tabular}

Low \& Medium: RIE-200L (Samco Inc.)

High: NLD-800 (ULVAC Inc.)

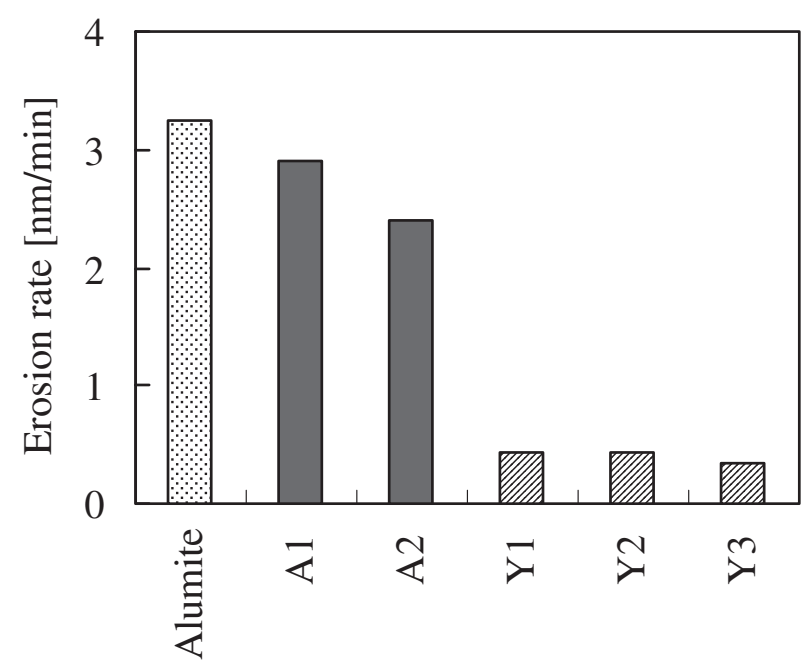

Fig. 2 Erosion rate of the alumite film and the spray coatings of alumina (A1, A2) and yttria (Y1-Y3) at the low plasma power $(80 \mathrm{~W})$.

estimated by measuring the step height between masked area and eroded area using stylus method (Alpha-step, KLA Tencor Co. Ltd., USA.). As a reference, alumite film was also tested. The erosion mechanism is also discussed through the micro-structural analysis.

\section{Results and Discussion}

3.1 Plasma erosion test at the low plasma power $(80 \mathrm{~W})$

Figure 2 shows the erosion rate of the alumite film and the plasma sprayed coatings of alumina and yttria at the low plasma power $(80 \mathrm{~W})$. In this figure, the low erosion rate means the high durability against the $\mathrm{CF}_{4} / \mathrm{O}_{2}$ plasma (highly anti-plasma erosion resistance). The alumite film with the erosion rate of $3.2 \mathrm{~nm} / \mathrm{min}$ has the lowest durability at the low plasma power and the durability of alumina coatings is slightly superior to the alumite film. The durability of the A2 coating, which has been prepared using smaller sized powder, is superior to that of the A1 coating. The yttria coatings, whose erosion rates are approximately $0.4 \mathrm{~nm} / \mathrm{min}$, have best anti-plasma erosion resistance. Also, it has been found that both the primary-particle diameter and the average diameter of the agglomerated-and-sintered powder barely affect the anti-plasma erosion resistance.

SEM images of the surface of the specimens are shown in Fig. 3. The alumite film, alumina (A2) and yttria (Y2) coatings are Figs. 3(a), (b), 3(c), (d) and 3(e), (f), respec- tively. The images in Figs. 3(a), (c), (e) are the surfaces before the erosion test and Figs. 3(b), (d), (f), (g), (h) are those after the test. Figures $3(\mathrm{~g})$ and $(\mathrm{h})$ are the yttria coatings of Y1 and Y3 after the test, respectively. The alumite film is eroded preferentially near the crack region as shown in Fig. 3(b) and homogeneous erosion is observed at another region. As for the plasma sprayed alumina (A2) coating, irregular surface is observed in Fig. 3(d) suggesting that inhomogeneous erosion. When the inter- and intra-granular regions, in other words, splat boundaries and inside of the splats, are compared in the spray coating, it is considered that the defect density, such as pores, impurities and crystal imperfectness, is higher in the inter-granular regions in general. This high defect density may degrade the antiplasma-erosion resistance at the inter-granular regions. In intra-granular regions, on the other hand, the defect density is considered to be lower that retains the highly anti-plasma erosion resistance. Because of inhomogeneous defect density distribution in the coating, the surface of alumina coating may become rough. Yttria coatings have smooth surface except for inter-granular regions, where the defect density is also considered to be higher resulting in preferential erosion. At intra-granular regions, the surface morphology between alumina and yttria is apparently different. Highly anti-plasma erosion resistance of yttria may result in such a smooth eroded surface. Within the yttria coatings in Figs. 3(f), (g), (h), the morphological difference between these eroded surfaces is unclear showing that no or little influence on the primary-particle size in this plasma erosion condition.

\subsection{Plasma erosion tests at medium $(175 \mathrm{~W})$ and high $(800 \mathrm{~W})$ plasma power}

Erosion rates of the plasma sprayed coatings, the alumite film and sintered-bulk ceramics at the both medium $(175 \mathrm{~W})$ and high $(800 \mathrm{~W})$ plasma power is shown in Fig. 4. The erosion rates of all specimens are apparently increased with increasing the plasma power from 80 to $800 \mathrm{~W}$ showing that the high plasma power decreases the lifetime of the shielding parts. At all erosion conditions, the alumite film shows the lowest durability. Similarly, yttria specimens have the highest durability and alumina specimens show medium performance. The difference of the erosion rate between the alumite film and the alumina spray coatings is relatively small at both conditions of 80 and $175 \mathrm{~W}$, and becomes larger with increasing plasma power $(800 \mathrm{~W})$. This tendency is same as between the alumite film and yttria coatings. Although the plasma power in commercial fabrication processes of the Si and LCD devices is selected flexibly, the plasma power tends to be increasing for advanced microfabrications. Considering that this trend, replacement from the alumite to ceramic coatings for the shielding parts is reasonable.

The sintered-bulk alumina (A3) shows higher anti-plasma erosion resistance compared to the alumina coatings (A1 and A2). Within yttria specimens, the bulk (Y5) is better than the yttria coatings similarly. It is considered that the difference is caused by the difference in the quality of the sintered-bulk and the coating. The quality of the bulk materials is generally superior to that of the spray coatings, such as crystal structure, its uniformity, porosity, apparent density, defect 
(a) alumite, before
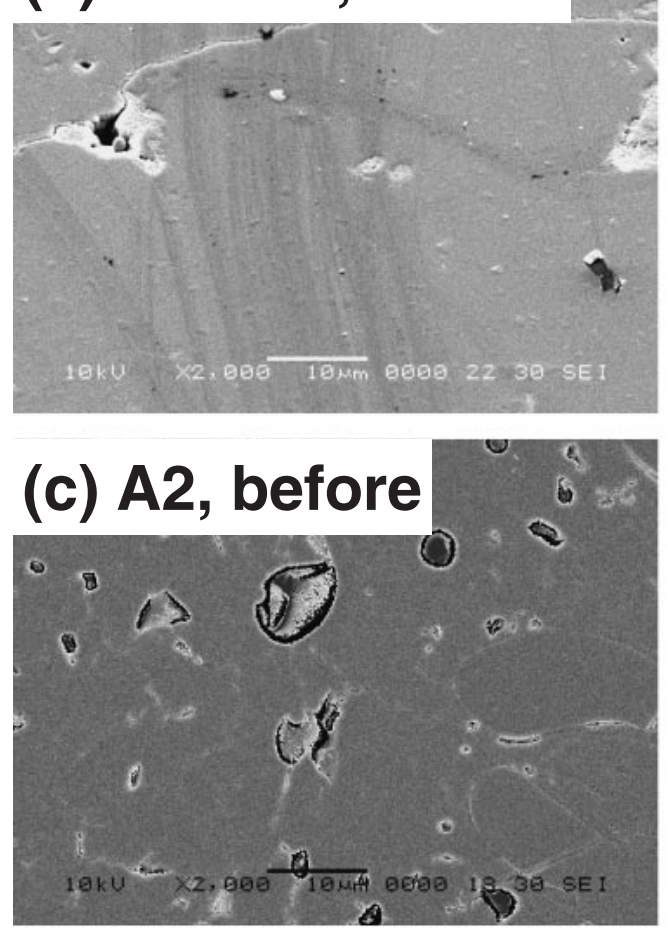

(e) Y2, before
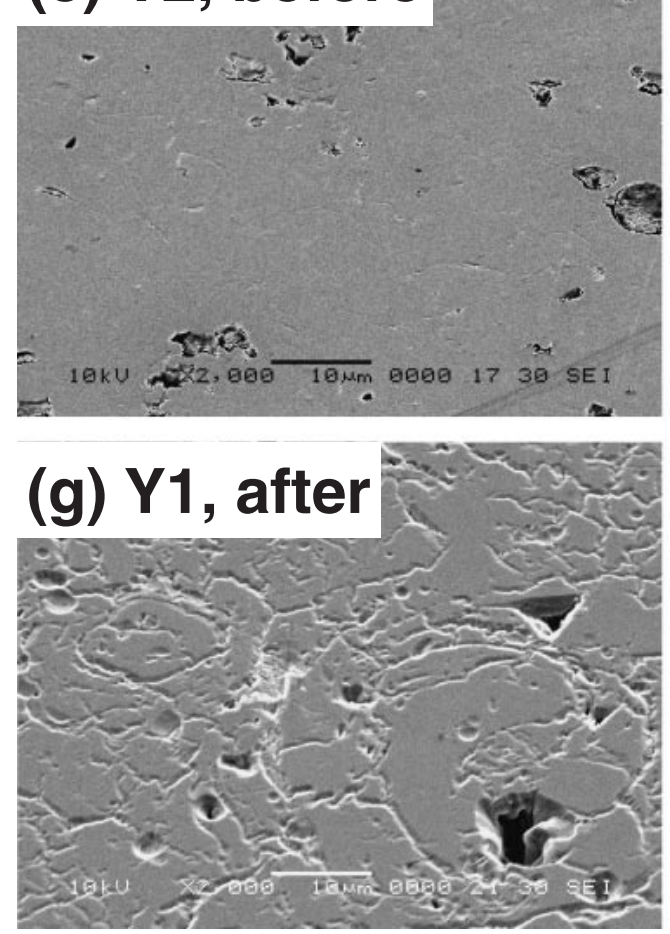

\section{(b) alumite, after}
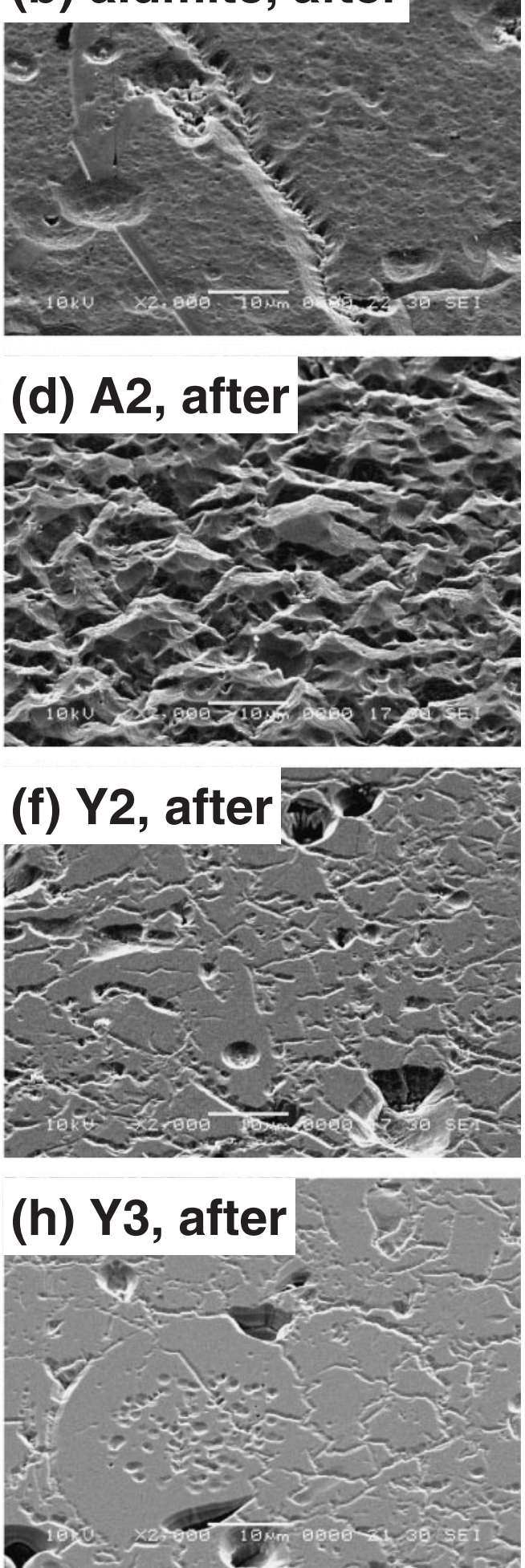

Fig. 3 SEM images of the surface of the specimens (a), (c), (e) before and (b), (d), (f), (g), (h) after the plasma erosion test at low power $(80 \mathrm{~W})$. The alumite film, alumina (A2) and yttria (Y2) coatings are (a, b), (c, d), and (e, f), respectively. Yttria coatings of Y1 and Y3 after the test are $(\mathrm{g})$ and $(\mathrm{h})$, respectively.

density, mechanical properties and residual stress. This may affect the performance of the anti-plasma erosion resistance. Although the bulk alumina has higher anti-plasma erosion resistance than the alumina coatings, yttria coatings have much higher resistance than all alumina specimens. This shows that reactivity of yttria against $\mathrm{CF}_{4} / \mathrm{O}_{2}$ plasma is lower than that of alumina. In the RIE by $\mathrm{CF}_{4}$ plasma, radicals and ions act multiply to etch the specimens. One of the possible erosion mechanisms of alumina and yttria by $\mathrm{CF}_{4}$ is chemical etching and its reaction formulas are as follows. 


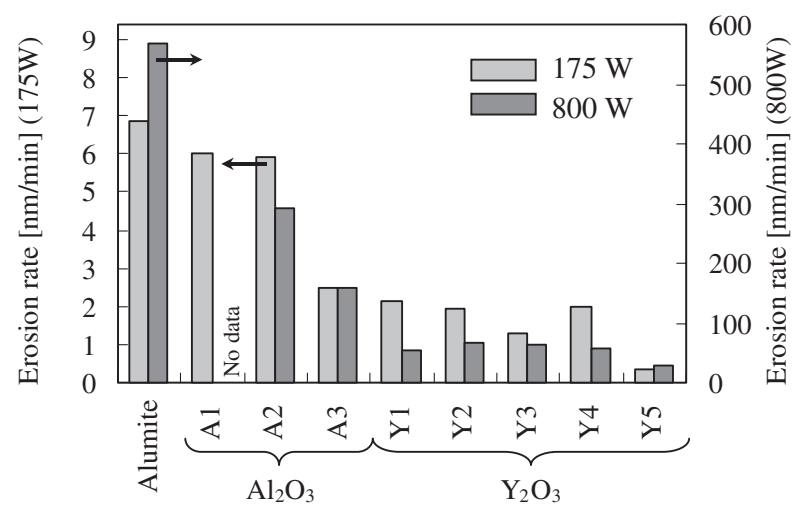

Fig. 4 Erosion rates of the alumite film, the spray coatings and sinteredbulk ceramics at the both medium $(175 \mathrm{~W})$ and high plasma power $(800 \mathrm{~W})$.

$$
\begin{aligned}
& \mathrm{Al}_{2} \mathrm{O}_{3}+3 \mathrm{CF}_{2}{ }^{*} \rightarrow 2 \mathrm{AlF}_{3} \uparrow+3 \mathrm{CO} \uparrow \\
& \mathrm{Y}_{2} \mathrm{O}_{3}+3 \mathrm{CF}_{2}{ }^{*} \rightarrow 2 \mathrm{YF}_{3} \uparrow+3 \mathrm{CO} \uparrow
\end{aligned}
$$

As for the reaction formulas, oxides become fluorides, and followed the vaporization of the fluorides. Highly stable yttria and low evaporation rate of yttrium fluoride may cause the low erosion rate of yttria. Another possible mechanism is the resistance against sputtering of $\mathrm{CF}_{3}{ }^{+}$or oxygen ions, which is called physical etching. Generally, low weight material is easily sputtered by ion beams. Atomic mass of yttrium is higher compared to that of aluminum, and yttria $\left(5.01 \mathrm{~g} / \mathrm{cm}^{3}\right)$ has higher density than alumina $\left(3.96 \mathrm{~g} / \mathrm{cm}^{3}\right)$. This may result in the lower sputtering rate of yttria specimens against the ions. Therefore, we can say that yttria is a promising material for the shielding parts in semiconductor production equipments compared to alumina. However, the yttria coatings (Y1-Y4) are still inferior to the sintered yttria (Y5) presently. This means that control of granular shape in the yttria coating by optimizing spray powders and spray conditions will improve the coating quality resulting in much higher anti-plasma erosion resistance almost same as sintered-bulk yttria. In order to find the way to improve the performance of the yttria coating, Y1-Y5 specimens are investigated in detail as follows.

\subsection{Comparison of yttria coatings and sintered yttria}

As mentioned previously, the effects of both the primaryparticle size and the average diameter of the agglomeratedand-sintered powder on the anti-plasma erosion resistance are little at low plasma power $(80 \mathrm{~W})$. When the plasma power increases to 175 or $800 \mathrm{~W}$, on the other hand, these effects are clearly seen. Figure 5 shows the erosion rates of the plasma sprayed coatings and the sintered-bulk of yttria, which are selected from the Fig. 4 to clarify the effects. The Y3 coating, which is prepared using agglomerated-and-sintered powder with a largest primary-particle size of $5.3 \mu \mathrm{m}$, has best durability at the medium plasma power $(175 \mathrm{~W})$ than the other coatings. The durability tends to decrease with decreasing the primary-particle size. The erosion rate of the Y3, best within the coatings, is still about 4 times as high as that of sintered yttria (Y5) showing that the low performance of the spray coatings. In contrast, at the higher plasma power

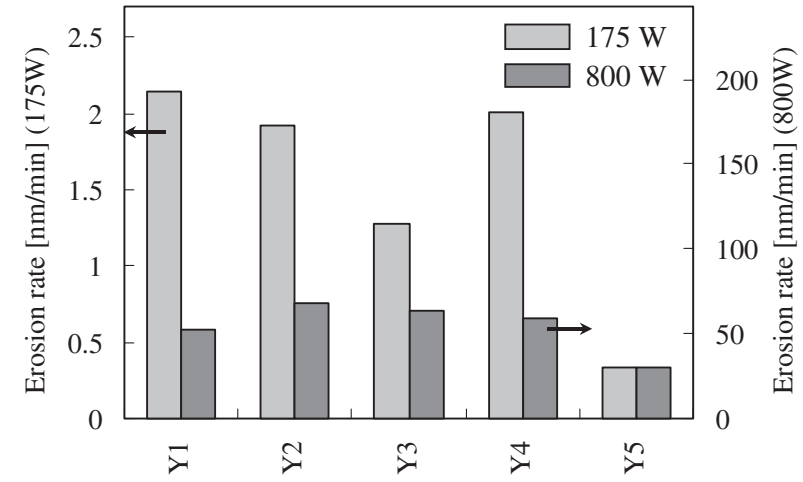

Fig. 5 Erosion rates of the spray coatings (Y1-Y4) and the sintered-bulk (Y5) of yttria at the both medium $(175 \mathrm{~W})$ and high plasma power $(800 \mathrm{~W})$.

$(800 \mathrm{~W})$, the durability becomes better with decreasing primary-particle size. The Y1 coating prepared using agglomerated-and-sintered powder with a smallest primaryparticle size $(0.6 \mu \mathrm{m})$ shows best performance within the coatings. The pink colored Y1 coating is simply considered to have low anti-plasma erosion resistance at first because of change in the color suggesting that degradation of characteristics of yttria. However, although its mechanism is unclear, the anti-plasma-erosion resistance is higher than the white colored coatings contrary. Further, the difference of the erosion rate between the coating and sintered-bulk becomes small with increasing plasma power. The erosion rate of the Y1 is only about 2 times as high as that of Y5. In order to clarify the effect of the plasma power on the durability of the yttria specimens, microstructure of the eroded surfaces is investigated by SEM observation as shown in Fig. 6. When the plasma power increases to $175 \mathrm{~W}$, rough surfaces are generated as shown in Figs. 6(a), (c) and (e) compared to the images $(80 \mathrm{~W})$ in Figs. $3(\mathrm{~g})$, (f) and (h), respectively. The rough surface suggests that the coatings are eroded almost similarly at both inter- and intra-granular regions. It is easy to consider that radicals and ions become much active with increasing plasma power resulting in considerable erosion at intra-granular regions. In comparison to the Y1, Y2 and Y3 coatings, surface morphology of the $\mathrm{Y} 3$ coating (e) with best durability is smooth. High resistant coating shows smoother surface after plasma erosion test due to lower volume of erosion.

Morphological feature of the sintered yttria at $175 \mathrm{~W}$ (Fig. 6(i)) is apparently different from that of the coatings at the same power in Figs. 6(a), (c), (e) and (g), where spherical pits are observed. Since sintered oxide ceramics, which is produced at high temperature with long-term duration in air or oxygen, has higher intergranular bonding strength than the spray coatings in general, the defect density is relatively lower at inter-granular regions. The lower defect density may avoid the preferential erosion at the inter-granular regions and cause the spherical pits. In spite of no observation of spherical pits on the spray coatings, the erosion at the intergranular region with a lower defect density seems to be almost same as the sintered-bulk yttria. From the formation of rougher surfaces at the high plasma power $(800 \mathrm{~W})$ shown in Figs. 6(b), (d), (f), (h) and (j), erosion at the intra-granular region becomes much considerable that may be almost 

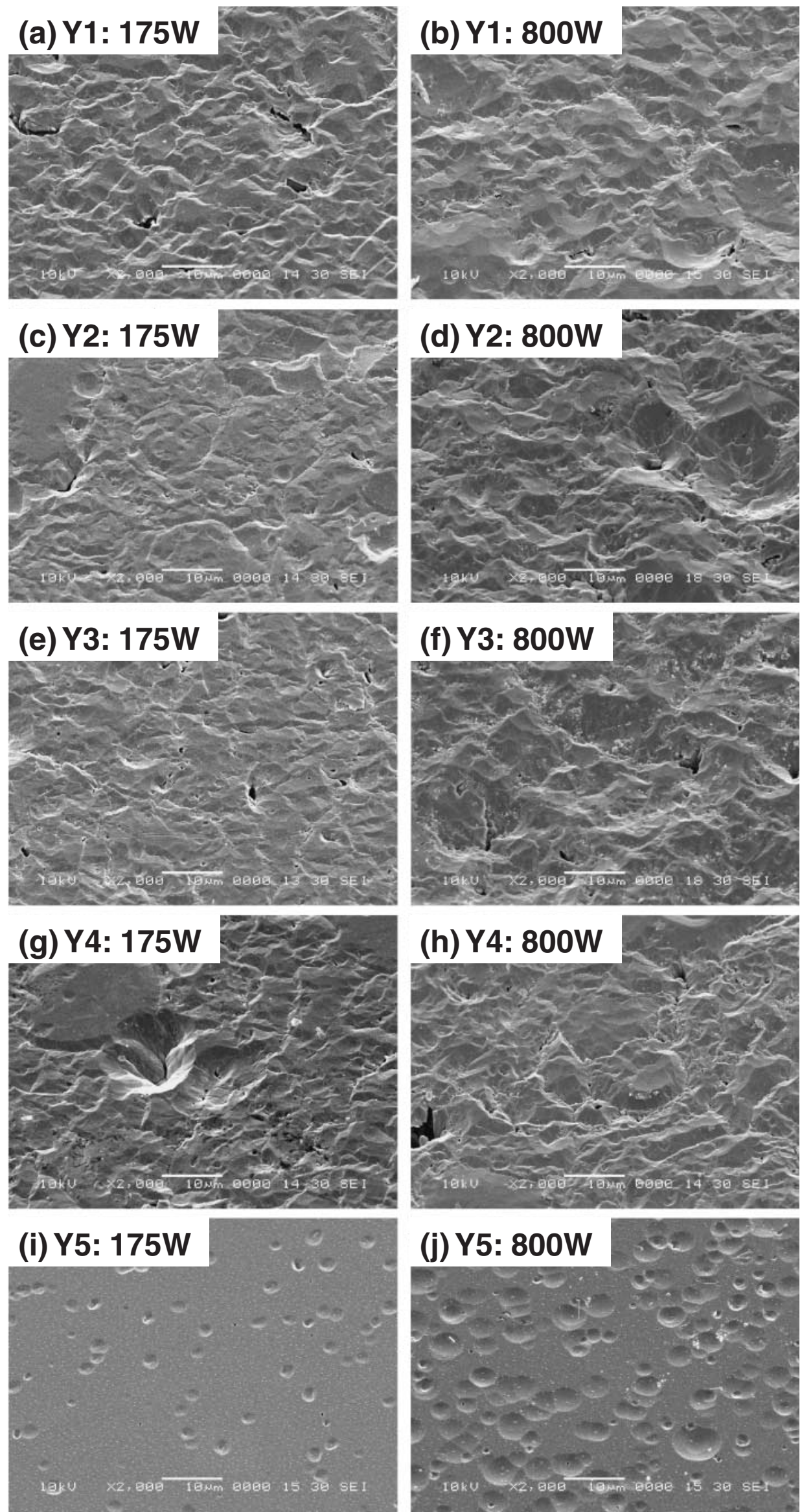

Fig. 6 SEM images of the surface of the yttria specimens after the plasma erosion tests. (a, b), (c, d), (e, f), and (g, h) are Y1, Y2, Y3 and Y4 coatings, respectively. (i) and (j) are sintered yttria (Y5). Plasma power at the tests are (a), (c), (e), (g), (i) 175 and (b), (d), (f), (h), (j) $800 \mathrm{~W}$, respectively. 
comparable to at the inter-granular region. As for the spray coatings, the surface morphology becomes much irregular. Pits size and its density are increased on the sintered-bulk. The major difference between the coating and the sinteredbulk is considered to be the quality of the inter-granular regions. Since the erosion at low power is dominant at the inter-granular regions, it is considered the erosion rate of the coatings becomes much higher than that of the bulk. In contrast, the effect of the intra-granular region becomes considerable with increasing plasma power. This may cause that the difference of the durability between the coating and the bulk becomes smaller with increasing plasma power.

It is assumed that the defect density at the inter-granular region in the coating is lower when the larger primary particle is used, such as Y3 coating, because of lower surface area of the powder. This lower defect density at the inter-granular region may cause the relatively higher erosion resistance compared to Y1 and Y2 coatings at medium plasma power $(175 \mathrm{~W})$. Contrary, it is considered that the smaller primary particle, such as $\mathrm{Y} 1$, is easily melted during the spraying process and inclusion of insufficient molten particle decreases that result in the improvement of the quality at the intragranular region in the coating. Therefore, higher erosion resistance of Y1 coating at high plasma power $(800 \mathrm{~W})$ may be produced.

\section{Conclusions}

$\mathrm{CF}_{4} / \mathrm{O}_{2}$ plasma erosion test by reactive ion etching (RIE) system revealed the following conclusions:

(1) Yttria coatings have higher anti-plasma erosion resistance compared to alumina coatings and the conventional alumite film. This shows that yttria is a promising material for the shielding parts in semiconductor and liquid-crystal-display production equipments.

(2) The yttria coating prepared using agglomerated-and- sintered powder with a larger primary-particle size has higher durability at medium plasma power $(175 \mathrm{~W})$. On the other hand, the coating by agglomerated-andsintered powder with a smaller primary-particle showed the best performance at high plasma power $(800 \mathrm{~W})$.

(3) It is found that the erosion at low plasma power $(80 \mathrm{~W})$ is dominant at the inter-granular regions in the yttria coating and the erosion at the intra-granular regions becomes considerable with increasing plasma power, clarified by microscopic observations.

(4) Although sintered-bulk yttria is superior to the yttria coatings at every plasma power conditions, the difference of the performance between the bulk and the coatings becomes smaller with increasing plasma power.

\section{Acknowledgement}

A part of this work was conducted in AIST NanoProcessing Facility, supported by "Nanotechnology Support Project" of the Ministry of Education, Culture, Sports, Science and Technology (MEXT), Japan.

\section{REFERENCES}

1) Y. Kobayashi: The Proc. 37th Seminar on High-Temperature Ceramics, (The Ceramics Society of Japan, 2005) p. 1-7 (in Japanese).

2) R. Ohtsuki: Publication of Japanese patent application, 2001-226773.

3) Status and perspective of thermal spray markets 2004, Digital Research Institute Inc. (Nagoya, Japan), 2004, p. 20-33 (in Japanese).

4) R. V. Gansert: The Proc. International Thermal Spray Conference 2003, (ASM International, 2003) p. 159-161.

5) T. Tsukatani and Y. Takai: Publication of Japanese patent applications, 2002-80954 (2002).

6) J. Kitamura, I. Aoki and Y. Sugiyama: Application for Japanese patents (2005). 